\title{
Norepinephrine exposure restrains endometrial decidualization during early pregnancy
}

\author{
Jiju Wang, Yunhui Tang, Songcun Wang, Liyuan Cui, Dajin Li and Meirong Du
}

Laboratory for Reproductive Immunology, NHC Key Lab of Reproduction Regulation (Shanghai Institute of Planned Parenthood Research), Shanghai Key Laboratory of Female Reproductive Endocrine Related Diseases, Hospital of Obstetrics and Gynecology, Fudan University Shanghai Medical College, Shanghai, People's Republic of China

Correspondence should be addressed to D Li or M Du: djli@shmu.edu.cn or dmrlq1973@sina.cn

\begin{abstract}
Previous studies have focused on the role of norepinephrine on arrhythmias, generalized anxiety disorder, and cancer. This study aimed to investigate the effect of norepinephrine on endometrial decidualization. Artificial decidualization and norepinephrine-treated mice were established in vivo. In vitro, human endometrial stromal cells were treated with MPA and cAMP to induce decidualization. Decidual markers and important signaling molecules during decidualization were detected using quantitative real-time PCR and Western blot. RNA sequencing was performed to determine related signaling pathways. Exposure to excess norepinephrine significantly restricted the induced expression of decidualized markers Dtprp, BMP2, WNT4, and Hand 2 in mice. In vitro, $10 \mu \mathrm{M}$ norepinephrine markedly downregulated the expressions of prolactin, IGFBP1, and PLZF, which are the specifical markers of decidual stromal cells during decidualization. The gene set enrichment analysis showed a significant enrichment in neuroactive ligand-receptor interactions of norepinephrine treatment group. The $\alpha 1$ b-adrenergic receptor expression was upregulated by norepinephrine. Interestingly, norepinephrine did not inhibit the expression of IGFBP1 in endometrial stromal cells after silencing $\alpha 1 \mathrm{~b}$-adrenergic receptor, while significantly suppressed the induced decidualization with overexpression of $\alpha 1 \mathrm{~b}$-adrenergic receptor. When $\alpha 1$-adrenergic receptor was activated, endometrial p-PKC was significantly increased under post-treatment with norepinephrine in vivo and in vitro. In addition, norepinephrine treatment inhibited embryo and fetal development using a normal pregnancy model. Therefore, norepinephrine exposure inhibited endometrial decidualization through the activation of the PKC signaling pathway by upregulating $\alpha 1$ b-adrenergic receptor. Our study could explain some female reproductive problems due to stress and provide some novel strategies for this disorder.
\end{abstract}

\section{Key Words}

- norepinephrine

- endometrial decidualization

- stress

- $\alpha 1$ b-adrenergic receptors

\section{Introduction}

In modern society, young women face extensive social and life pressures. Long-term sustained or acute stress can lead to a pathological state that impacts the reproductive system (Frazier et al. 2018, Wang et al. 2019). Physiological or psychological stressors that induce the sympathetic nervous system's 'fight-or-flight' response can rapidly increase the norepinephrine (NE) level (Tank \& Lee Wong 2015). However, the effect of $\mathrm{NE}$ on reproductive toxicity in early pregnancy had not been reported. 
Endometrial decidualization is an important step in embryonic development and fetal growth. In particular, this process is critical in establishing maternal-fetal communication and provide a good nutritional environment for embryonic development (Ng et al. 2020). Deficient decidualization can cause restricted embryonic growth (Schatz et al. 2016). Analyzing the regulation of NE on decidualization will help us understand the influence of stress on endometrial decidualization and fetal growth.

In early pregnancy, endometrial stromal cells (ESCs) undergo extensive proliferation and differentiation and transform into secretory decidual stromal cells, leading to decidualized endometrium. The decidual/ trophoblastic PRL-related proteins (Dtprp) (Kimura et al. 2001), Bmp2 (Li et al. 2007), Wnt4 (Li et al. 2013), and Hand2 (Murata et al. 2020) are well-known markers for uterine stromal differentiation during decidualization in mice. In vitro, in response to P4-mediated activation of cyclic adenosinemonophosphate (cAMP) signaling, human ESCs undergo vast expansion and differentiation. Prolactin (Eyal et al. 2007), IGFBP1 (Tabanelli et al. 1992), and promyelocytic leukemia zinc finger encoded by Zbtb16 (PLZF) (Brosens \& Gellersen 2006) have been found as well-established stromal differentiation markers during ESC decidualization. Besides, forkhead box O1 (FOXO1) (Buzzio et al. 2006, Park et al. 2016) and CCAAT enhancer-binding protein $\beta$ (C/EBP $\beta)$ (Mantena et al. 2006, Wang et al. 2010, Tamura et al. 2018) are core decidual transcription factors that control cell cycle exit of ESCs in response to differentiation signals, activating expression of decidual marker genes, such as PRL and IGFBP1.

In this study, we hypothesized that NE inhibited endometrial decidualization. To test this hypothesis, a series of in vivo and in vitro experiments were conducted with NE to mimic stress. Sesame oil was injected into the uterine horn of mice to induce endometrial decidualization (Andrade et al. 1996); therefore, its influence on the embryo may be ruled out. Further, it was intraperitoneally injected with NE to observe its effect on decidualization. In vitro, primary stromal cells were isolated from human endometrium, treated them with cAMP and medroxyprogesterone acetate (MPA) for $48 \mathrm{~h}$ to induce decidualization (Salsano et al. 2017), and simultaneously added with NE to observe its effect on decidualization of human ESCs. Our results demonstrated that NE treatment inhibited endometrial decidualization in early pregnant mice through the activation of the PKC signaling pathway by upregulating $\alpha 1 \mathrm{~b}$-adrenergic receptor ( $\alpha 1 \mathrm{~b}-\mathrm{AR})$. This study would provide treatment ideas for endometrial decidualization disorders caused by stress.

\section{Materials and methods}

\section{Animals and tissue collection}

C57 female and male mice, aged 8-10 weeks, were obtained from the Shanghai Laboratory Animal Center (Shanghai, China). All mice were caged at a controlled temperature of $22-25^{\circ} \mathrm{C}$ under a $14 \mathrm{~h}$ light: $10 \mathrm{~h}$ darkness photoperiod. The Human Research Ethics Committee of Obstetrics and Gynecology Hospital of Fudan University approved the study protocol. To observe the effects of NE on normal female pregnancy, adult female mice were allowed to mate with fertile males to achieve pregnancy. The day on which sperms were detected in the vaginal smear was defined as gestational day 0.5 (GD 0.5) of pregnancy. Pregnant female mice were divided into two groups according to the dosage in previous articles (Wang \& Yang 2007): the $\mathrm{NE}$ group was injected with $5 \mathrm{mg} / \mathrm{kg} \mathrm{NE}$ intraperitoneally daily from GD 5.5 for three consecutive days, whereas the control group was injected with the same volume of PBS. Uterine tissue was collected from each pregnant mouse on GD 8.5 and GD 13.5. The number and size of the embryos were recorded.

\section{Artificial decidualization induction}

To examine the effects of $\mathrm{NE}$ on endometrial decidualization, adult female mice were allowed to mate with vasectomized adult males to achieve pseudopregnancy. Artificial decidualization was induced by infusing $25 \mu \mathrm{L}$ of sesame oil intraluminally into one uterine horn on GD 3.5 of pseudopregnancy; the contralateral uninjected horn was considered as the control (Andrade et al. 1996). Pseudopregnant female mice were divided into two groups (NE and control) treated identically with that of the pregnant mice. NE (5 $\mathrm{mg} / \mathrm{kg}$ ) was injected daily for 3 days, beginning at $24 \mathrm{~h}$ after the oil stimulation or saline injection that served as the control. These mice were sacrificed at GD 7.5 of pseudopregnancy. Decidualization was confirmed by weighing the uterine horns and histological examination of the uterine sections.

\section{Culture of human ESCs}

Human endometrial tissue was obtained from 12 healthy volunteers of reproductive age. The Ethics Committee of the Obstetrics and Gynecology Hospital of Fudan University approved the study protocols. All participants provided written informed consents. Human ESCs were 
isolated as previously described (Li et al. 2011). Briefly, the endometrial tissue was minced and digested with collagenase type IV (0.1\%; Sigma-Aldrich) and 15 units/mL of deoxyribonuclease (Sigma-Aldrich) for $30 \mathrm{~min}$ at $37^{\circ} \mathrm{C}$. The dispersed cells were passed through a $40-\mu \mathrm{m}$ filter (BD Biosciences). After centrifugation at $800 \boldsymbol{g}$ for $5 \mathrm{~min}$, ESCs were resuspended and maintained in DMEM/F-12 containing 10\% fetal bovine serum (FBS; Gemini, Calabasas, USA), $100 \mathrm{IU} / \mathrm{mL}$ penicillin (Sigma), $100 \mu \mathrm{g} / \mathrm{mL}$ streptomycin (Sigma), and $1 \mu \mathrm{g} / \mathrm{mL}$ amphotericin B (Sangon, Shanghai, China) at $37^{\circ} \mathrm{C}$ in $5 \% \mathrm{CO}_{2}$. The cultured stromal cells were $95 \%$ pure, as determined using vimentin staining.

\section{Induction of in vitro decidualization and cell viability assay}

To induce decidualization, approximately $3 \times 10^{5}$ cells were plated per well in 6-well culture plates. After reaching 70-80\% confluency, cells were treated with $1 \mu \mathrm{M}$ MPA (Sigma) and $0.5 \mathrm{mM} \mathrm{N6}, 2$ '-O-dibutyryladenosine cAMP sodium salt (Sigma) for 48 h (Salsano et al. 2017). Cell viability was assessed using the Cell Counting Kit-8 (CCK-8) assay (Dojindo-Japan, CK04).

\section{Cell transfection}

Transfection of $\alpha 1 b$-AR small-interfering (si)RNA. Human ESCs were transfected with $\alpha 1 b-A R$ siRNA or nontargeting siRNA (N.C siRNA) (GenePharma, Shanghai, China) diluted in transfection medium. The sequence of $\alpha 1 \mathrm{~b}-\mathrm{AR}$ siRNA was as follow: sense 5'-GGAGUCAUGAAGGAGAUG UTT-3', antisense 5'-ACAUCUCCUUCAUGACUCCTT-3'. Transfection was performed using the Lipofectamine TM 3000 reagent (Invitrogen). The knockdown efficiency of $\alpha 1 \mathrm{~b}-\mathrm{AR}$ in human ESCs was assessed using Western blotting.

Transfection of $\alpha 1 \mathrm{~b}-\mathrm{AR}$ overexpression plasmid. All plasmids were purchased from PPL (Genebio Technology, lnc, Nanjing, China): $\alpha 1 \mathrm{~b}-\mathrm{AR}$ overexpression plasmid pLenti-CMV- $\alpha 1 b-A R-G F P-P u r o$ (Flag- $\alpha 1 b-A R$ ) with a corresponding negative control (pLenti-CMV-GFP-Puro, vector). The aforementioned plasmids were transfected into human ESCs using the Lipofectamine TM 3000 reagent (Invitrogen) according to the manufacturer's protocol. At $48 \mathrm{~h}$ post-transfection, the medium was gently exchanged to the new neuronal culturing medium supplemented with cAMP and MPA. After $48 \mathrm{~h}$, cells were harvested for Western blot analysis.

\section{mRNA sequencing data analysis}

To explore the NE mechanism affecting decidualization, mRNA sequencing on induced ESCs treated with or without NE was performed to determine related signaling pathways. Sequenced reads were aligned to the human reference genome using the STAR software package. Exons from all gene isoforms were merged to create one metagene. The number of reads falling in the exons of this metagene was counted using HTSeq-count, and differential expression analysis was conducted using DE-Seq. $P<0.05$ were considered as the significance threshold. Volcano Plot and Heatmap analysis of differential genes was performed using the online gene set enrichment analysis (GSEA) (Subramanian et al. 2005, Anders \& Huber 2010, Liu et al. 2020).

\section{Immunohistochemistry staining}

Decidual tissue sections were immunostained with primary antibodies against $\alpha 1 \mathrm{~b}-\mathrm{AR}$ (1:200, Abcam) overnight at $4^{\circ} \mathrm{C}$. On the next day, sections were incubated with a goat anti-rabbit (rabbit ABC detect kit, ZSBio, Beijing, China) secondary antibody at $37^{\circ} \mathrm{C}$ for $30 \mathrm{~min}$. Next, they were stained with 3,3'-diaminobenzidine (DAB) and counterstained with hematoxylin. Control sections were run concurrently with experimental sections using nonspecific rabbit IgG and were similarly pretreated. Nonspecific staining was not detected in the controls.

\section{Western blotting analyses}

Whole-cell protein extracts were prepared by lysing cells in radioimmunoprecipitation assay buffer supplemented with proteinase inhibitors (Beyotime Institute of Biotechnology, Haimen, China). The protein yield was quantified using the bicinchoninic acid protein assay. After denaturation, equal amounts of protein were separated by SDS-PAGE before the wet-transfer onto polyvinylidene difluoride membranes. Nonspecific binding sites were blocked by incubating membranes with 5\% BSA in trisbuffered saline with $0.1 \%$ Tween 20 (TBS-T) for $2 \mathrm{~h}$. Then, membranes were rinsed and incubated overnight at $4^{\circ} \mathrm{C}$ with the following primary antibodies diluted in blocking buffer (5\% BSA and 1× TBS-T): insulin-like growth factor binding protein 1 (IGFBP1, Abcam, ab181141), FOXO1 (Cell Signaling Technology, \#2880), CCAAT enhancer-binding protein beta (CEBPB, Cell Signaling Technology, \#90081), a1b-AR (Abcam, ab169523), a2b-AR (Abcam, ab151727), ß2-AR (Abcam, ab182136), GADPH 
(Abcam,ab8245), $\beta$-actin (Beyotime; AA128), protein kinase C (PKC) $\alpha$ (Abcam, ab32376), phospho (p)-PKC $\alpha / \beta$ II (Thr638/641) (Cell Signaling Technology, \#9375), AKT (Cell Signaling Technology, \#4691), and p-AKT (Ser473) (Cell Signaling Technology, \#4060). Primary antibodies were removed by washing the membranes three times in TBS-T. Then, these membranes were incubated for $1 \mathrm{~h}$ with horseradish peroxidase-conjugated secondary antibodies (Beyotime; A0208 and A0216). After three washes with TBS-T, immunopositive bands on blots were visualized using an enhanced chemiluminescence detection system (Merck Millipore).

\section{Quantitative real-time polymerase chain reaction (qRT-PCR)}

The total RNA from uterine tissues or ESCs was extracted with TRIzol reagent (Invitrogen) and then reverse-transcribed into the first strand complementary (c) DNA (Qiagen) according to the manufacturer's instructions. The synthesized cDNA was amplified with specific primers and detected with SYBR Green (Qiagen) using a PRISM 7900 Sequence Detection System (Applied Biosystems). Triplicate samples were examined in each condition. A comparative threshold cycle (CT) value was normalized for each sample using the $2^{-\Delta \Delta C T}$ method. Primer sequences used for qRT-PCR are shown in Table 1.

\section{Statistical analyses}

Prism 8 software (GraphPad) was used for all data analyses. All values are presented as means \pm s.E.M. determined from at least three independent experiments. Analysis of measurement data was performed with Student's unpaired two-tailed $t$-test, one-way ANOVA with Bonferroni's post hoc test or repeated measures ANOVA with Bonferroni's post hoc test. Analysis of count data was performed with Chi-square test. $P<0.05$ was considered statistically significant.

\section{Results}

\section{NE exposure during early pregnancy restrains artificially induced decidualization}

To assess the effects of NE on decidualization, an artificial decidualization model that excluded interference from embryo was used (Fig. 1A). At $96 \mathrm{~h}$ of oil stimulation, the deciduoma in the NE-treated group were much smaller than those in the control group (Fig. 1B). Decreased decidual tissue weight was observed following the $\mathrm{NE}$ exposure (Fig. 1B and C). As shown in Fig. 1D, HE staining of deciduoma confirmed the negative effect of NE on decidualization. We also detected the Dtprp, Bmp2, Wnt4, and Hand2 expressions, which are regarded as endometrial decidualization markers. Results in Fig. 1E, F, G and $\mathrm{H}$ showed that NE treatment inhibited the expression of all four molecules on GD 7.5 as compared with that of the control group (Fig. 1E, F, G and H). These results demonstrated that NE exposure restrained endometrium decidualization of mice.

\section{NE inhibits decidualization of human ESCs induced by CAMP and MPA in vitro}

Human ESCs were used to observe the effects of NE on decidualization process in vitro. First, the CCK-8 assay was used to determine whether treatment with $0.1-, 1-$, or $10-\mu \mathrm{M} N E$ affected cell viability. Compared to the control group, none of these $\mathrm{NE}$ concentrations affected cell viability (Fig. 2A).

To elucidate the effects of $\mathrm{NE}$ on stromal cell differentiation, cAMP and MPA were used to induce decidualization with or without $0.1-, 1-$, or $10-\mu \mathrm{M}$ NE. The expression of IGFBP1, a well-known decidualization marker (Tabanelli et al. 1992), was significantly increased after cotreatment of cAMP and MPA for $48 \mathrm{~h}$. However, in the presence of $10 \mu \mathrm{M} \mathrm{NE}$, the upregulated expression of IGFBP1 decreased significantly (Fig. 2B). qRT-PCR results showed that $10-\mu \mathrm{M} N E$ markedly downregulated

Table 1 Forward and reverse primer sequences used in qRT-PCR.

\begin{tabular}{lll}
\hline Gene & & Forward (5--3') \\
\hline Dtprp & & AAGAATGCCCTTCAGCGAGC \\
Bmp2 & GGGACCCGCTGTCTTCTAGT \\
Wnt4 & AGACGTGCGAGAAACTCAAAG \\
Hand2 & GCTACATCGCCTACCTCA \\
IGFBP1 & CGAAGGCTCTCCATGTCACCA \\
Prolactin & AAGCTGTAGAGATTGAGGAGCAAAC \\
Promyelocytic leukemia zinc finger, encoded by Zbtb16 (PLZF) & TCACATACAGGCGACCACC \\
& &
\end{tabular}

Reverse (5'-3')

AGCTGGTGGGTTTGTGACAT TCAACTCAAATTCGCTGAGGAC GGAACTGGTATTGGCACTCCT CCTTCTTCCTCTTCTCCTCT TGTCTCCTGTGCCTTGGCTAAAC TCAGGATGAACCTGGCTGACTA CTTGAGGCTGAACTTCTTGC https://joe.bioscientifica.com

https://doi.org/10.1530/JOE-20-0479 (c) 2021 Society for Endocrinology Published by Bioscientifica Ltd. Printed in Great Britain 
A

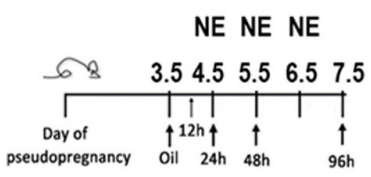

B

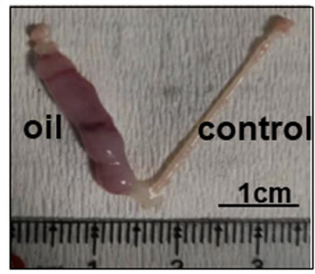

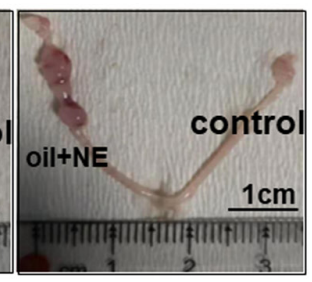

E

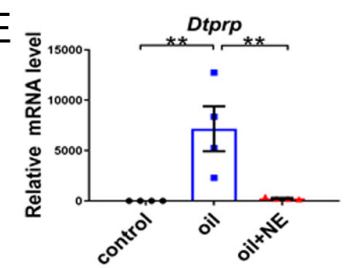

G

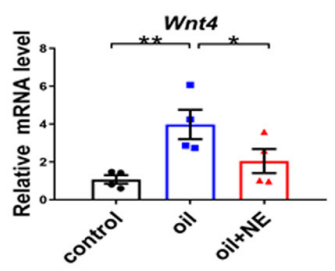

C

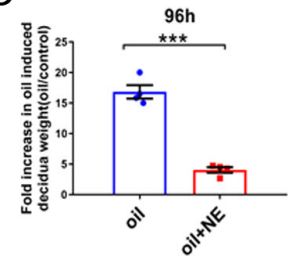

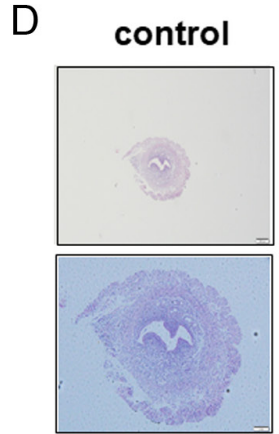
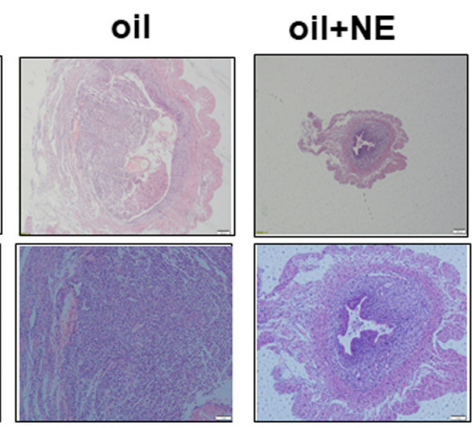

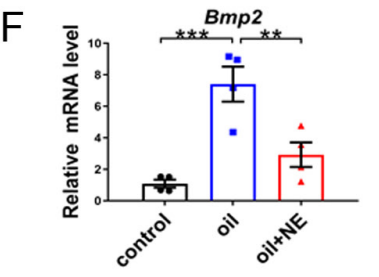

$\mathrm{H}$

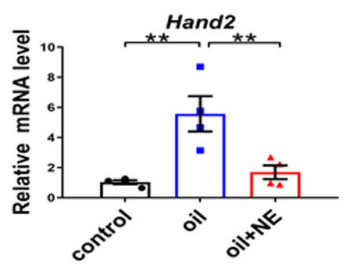

\section{Figure 1}

NE exposure during early pregnancy results in an artificial decidualization defect. (A) Artificial decidualization treatment and NE administration schedules. (B) Morphological differences of pseudopregnant uterus. Control, Non-decidualization; Oil, artificial decidualization; Oil + NE, artificial decidualization and NE injected intraperitoneally. (C) The fold increase after oil-induced decidual weight. Analysis was performed with Student's unpaired two-tailed $t$-test. $\star \star \star P<0.001$. (D) Paraffin sections of deciduoma and the control uterine horn were used for H\&E staining. The relative mRNA expressions of decidualization marker genes were examined by real-time PCR. (E) Dtprp. (F) Bmp2. (G) Wnt4. (H) Hand2. The values are normalized to the GAPDH expression level. Analysis was performed with one-way ANOVA and Bonferroni's post hoc test. $* P<0.05, * \star P<0.01, * \star \star P<0.001$. A full color version of this figure is available at https://doi.org/10.1530/JOE-20-0479.

the prolactin, IGFBP1, and promyelocytic leukemia zinc finger expression encoded by Zbtb16 (PLZF), the well-established stromal differentiation markers, during human ESC decidualization (Fig. 2C).

Finally, Western blots were used to analyze FOXO1 and CEBPB expressions, both of which are critical transcription factors for decidualization. The expression of both proteins was significantly increased $(P<0.05)$ after the induction of decidualization compared to cells without the induction of decidualization. Administration of $10 \mu \mathrm{M}$ NE recovers the FOXO1 and CEBPB levels upregulated by cAMP and MPA (Fig. 2D). These data suggested that NE inhibited decidualization of human ESCs induced by cAMP and MPA in vitro.

\section{NE inhibits decidualization by upregulating $\alpha 1 \mathrm{~b}-\mathrm{AR}$}

To explore the mechanism of NE affecting decidualization, RNA sequencing was performed to determine the related signaling pathways. Human ESCs were induced decidualization with or without NE treatment in vitro. Differentially expressed genes were demonstrated between two groups according to a heatmap (Fig. 3A). The GSEA showed a significant enrichment in neuroactive ligandreceptor interactions of NE treatment group, compared to the control group (Fig. 3B). Previous reports indicated that $\alpha 1 b-, \alpha 2 b-$, and $\beta 2$-AR were expressed in the mouse endometrium during decidualization (Wu et al. 2019). We found that $\alpha 1 b$-AR, $\alpha 2 b-A R$, and $\beta 2$-AR were also expressed in human ESCs; however, only $\alpha 1 b$-AR was upregulated after the NE treatment (Fig. 3C). Immunohistochemistry and Western blot were performed on artificially induced decidual tissue of mice, $\alpha 1 b-\mathrm{AR}$ expression in the $\mathrm{NE}$ treatment group was found to be significantly upregulated as compared with that in the control group (Fig. 3D and E). Pretreating ESCs with $10-\mu \mathrm{M}$ prazosin (an $\alpha 1 \mathrm{~b}-\mathrm{AR}$ inhibitor) (Murata et al. 1999) for $2 \mathrm{~h}$ reversed the NE effect on IGFBP1 expression (Fig. 3F). Then, we knocked down $\alpha 1 b-A R$ by transfecting ESCs with $\alpha 1 b-A R$ siRNA. After verifying the silence efficiency, we found that NE did not inhibit the IGFBP1 expression after knocking down $\alpha 1 b$-AR (Fig. 3G). Further, we over-expressed $\alpha 1 b$-AR in ESCs to observe the induced decidualization. As shown in Fig. 3H, IGFBP1 expression was downregulated under the 
A

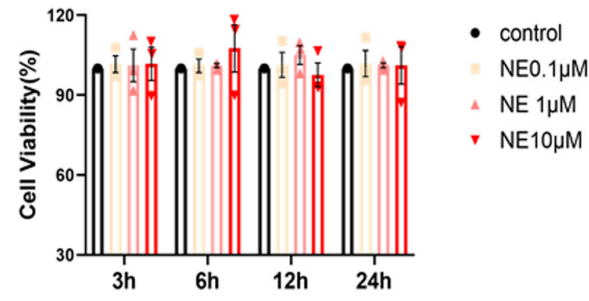

C

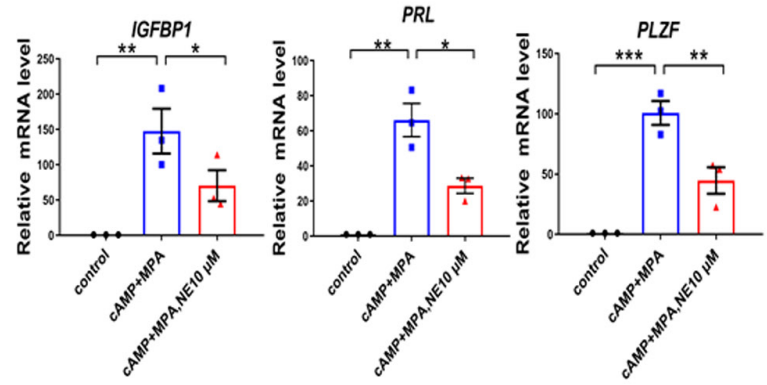

B
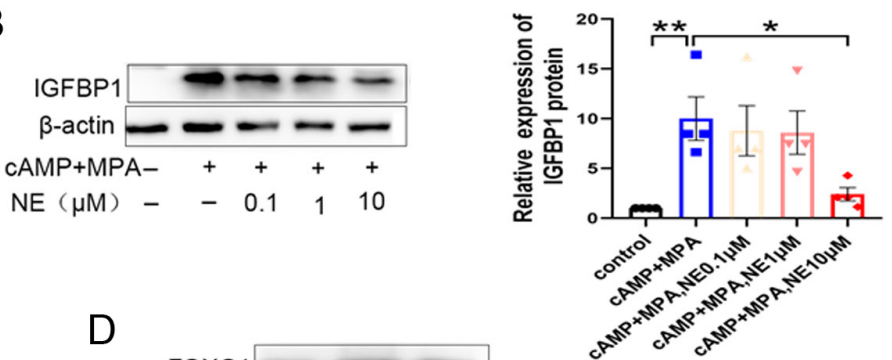

D
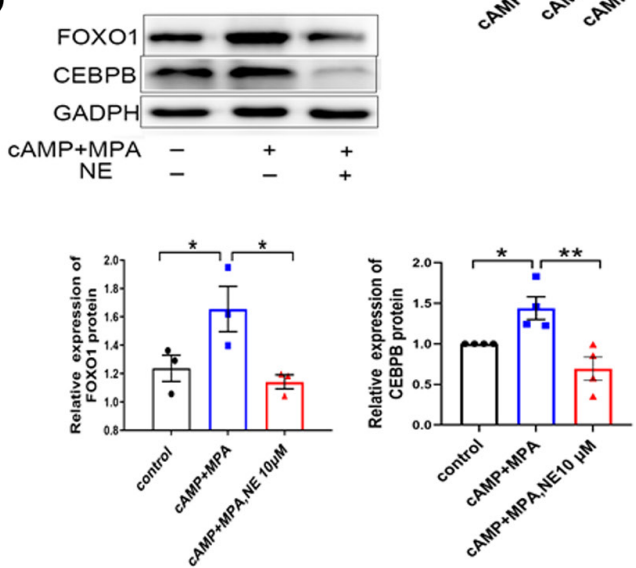

Figure 2

$\mathrm{NE}$ inhibits decidualization of human ESCs in vitro. (A) Effects of NE on stromal cell proliferation. After treatment with $0.1,1$, or $10 \mu \mathrm{M} \mathrm{NE} \mathrm{NE} \mathrm{for} 3 \mathrm{~h}, 6 \mathrm{~h}$, $12 \mathrm{~h}$ and $24 \mathrm{~h}$ in the presence of CAMP and MPA, stromal cells were analyzed by CCK8 assay. Analysis was performed with repeated measures ANOVA and Bonferroni's post hoc test. (B) The protein expressions of IGFBP1 in ESCs treated with CAMP and MPA with or without different concentrations of NE for $48 \mathrm{~h}$ were detected by Western blot. Analysis was performed with one-way ANOVA and Bonferroni's post hoc test. $* P<0.05, \star \star P<0.01$. (C) The mRNA level of well-established human stromal differentiation markers (IGFBPI, PRL and PLZF) in ESCs treated with cAMP and MPA with or without $10 \mu \mathrm{M}$ NE were examined by real-time PCR. Analysis was performed with one-way ANOVA and Bonferroni's post hoc test. $* P<0.05, * \star P<0.01, \star \star \star P<0.001$. (D) FOXO1 and CEBPB levels in ESCs treated with CAMP and MPA with or without $10 \mu \mathrm{M}$ NE were detected by Western blot. Data are the means \pm S.E.M.. Analysis was performed with one-way ANOVA and Bonferroni's post hoc test. $* P<0.05, * \star P<0.01$. A full color version of this figure is available at https:// doi.org/10.1530/JOE-20-0479.

decidualization induction after $\alpha 1 \mathrm{~b}-\mathrm{AR}$ overexpression as compared with the vector group. Altogether, these results indicated that NE inhibited decidualization via upregulating $\alpha 1 b-A R$.

\section{NE inhibits decidualization by activating PKC signaling pathway}

After activating $\alpha 1 \mathrm{~b}-\mathrm{AR}$, NE can further activate the PKC signaling pathway (Wang \& Ashraf 1998). Previous studies showed that PKC $\alpha$ expression was downregulated during decidualization (Shyu et al. 1997a,b). Consistent with this, p-PKC level in ESCs was found to be reduced after cAMP and MPA treatment, which were used to induce decidualization. Treatment with NE recovered the p-PKC level downregulated by cAMP and MPA (Fig. 4A). Although the phosphorylated level of AKT was also decreased significantly after cotreatment with cAMP and MPA for $48 \mathrm{~h}, 10 \mu \mathrm{M} \mathrm{NE}$ couldn't reverse the downregulated expression of p-AKT (Fig. 4A). Phorbol myristate acetate (a PKC pathway agonist, $200 \mathrm{nM}$ ) (Abdullah et al. 2003) significantly inhibited the IGFBP1 expression, whereas in the pretreatment with Ro 31-8220 mesylate (a PKC pathway Inhibitor, $0.25 \mu \mathrm{M}$ ) (Besson et al. 2019), NE could not inhibit IGFBP1 expression (Fig. 4B). Western blot analysis also revealed a significantly increased p-PKC level in the decidua tissue of the NE-treated mice, as compared with the control group (Fig. 4C). These results indicated that the ability of NE to inhibit decidualization was achieved by activating the PKC $\alpha$ signaling pathway after upregulating $\alpha 1 \mathrm{~b}-\mathrm{AR}$.

\section{NE inhibits fetal growth in the normal pregnancy mice}

To assess the impact of NE on pregnancy outcomes, the fetal growth and post-implantation mice injected with NE were examined (Fig. 5A). At GD 8.5, the average weight of the embryo implantation site (IS) was decreased in mice injected with NE compared with control mice (Fig. 5B 
A
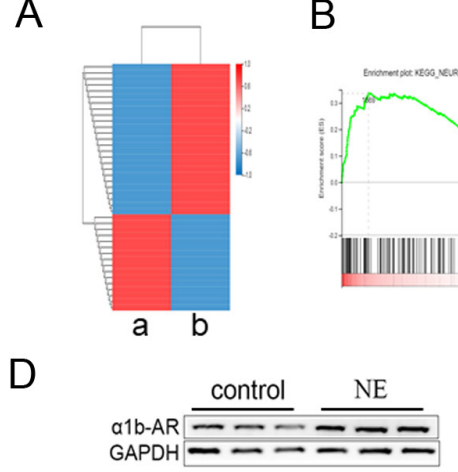

E

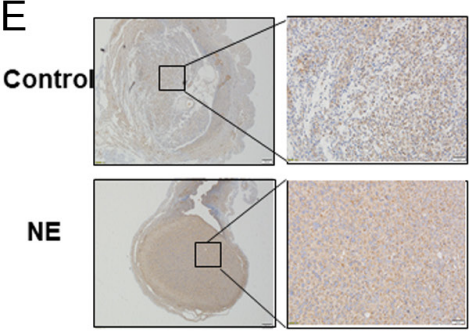

B

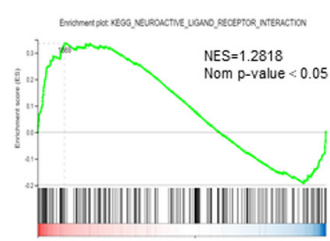

C
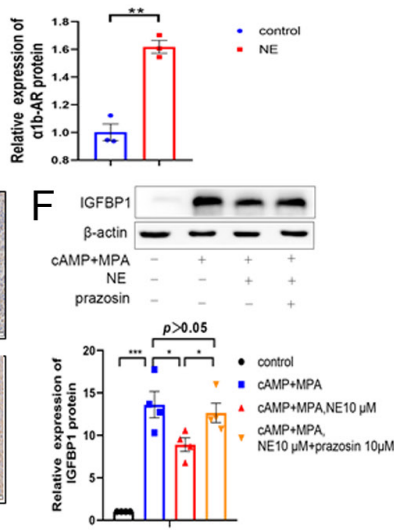
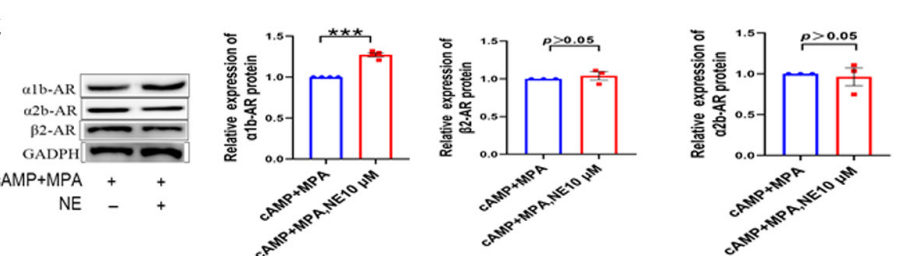

\section{$\mathrm{G}$}
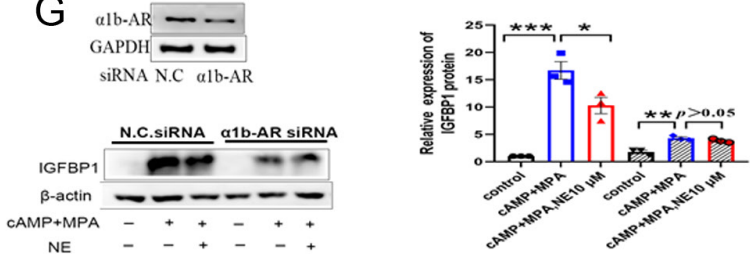

$\mathrm{H}$
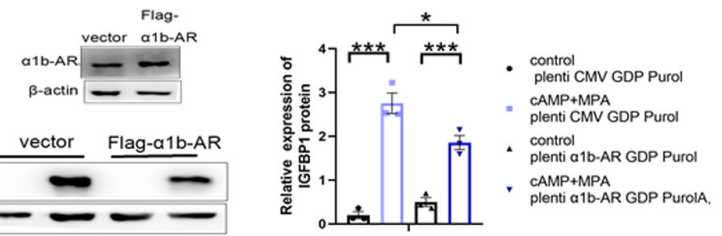

Figure 3

NE inhibits decidualization by upregulating $\alpha 1 \mathrm{~b}-\mathrm{AR}$. (A) Heatmap result of an unsupervised hierarchical clustering of genes that is significantly different $(P<0.01)$ in induced ESCS treated with or without NE. Each column represents a group, and each row represents a gene. The heatmap indicates the level of row normalized gene expression. Red, high expression; Blue, low expression; $a$, ESCs treated with cAMP and MPA for 48 h; b, ESCs treated with cAMP, MPA, and $10 \mu \mathrm{M}$ NE for $48 \mathrm{~h}$. (B) The gene set enrichment analysis of ESCs treated with CAMP and MPA with or without $10 \mu \mathrm{M}$ NE. The expression of $\alpha 1 \mathrm{~b}-\mathrm{AR}, \alpha 2 \mathrm{~b}-\mathrm{AR}$ and $\beta 2$-AR in ESCs was analyzed by Western blot (C). Analysis was performed with Student's unpaired two-tailed $t$-test. *** $P<0.001$. (D) The protein expressions of $\alpha 1 b-A R$ on artificially induced decidual tissue of mice between the NE treatment group and the control group were detected by Western blot. (E) Immunohistochemical analysis of $\alpha 1 \mathrm{~b}$-AR protein expression on artificially induced decidual tissue in mice. (F) The protein levels of IGFBP1 in ESCS after pretreatment with or without prazosin for $30 \mathrm{~min}$. Analysis was performed with one-way ANOVA and Bonferroni's post hoc test. * $P<0.05$, $\star \star \star P<0.001$. (G) Representative images of the protein levels of $\alpha 1 \mathrm{~b}$-AR after transfection with N.C siRNA or $\alpha 1 \mathrm{~b}-\mathrm{AR}$ siRNA. Western blot analysis of the protein levels of IGFBP1 as described. Analysis was performed with one-way ANOVA and Bonferroni's post hoc test. $* P<0.05, * \star P<0.01$, $* \star \star P<0.001$. (H) Overexpression of Flag- $\alpha 1$ b-AR was sufficient to decrease IGFBP1 at the protein level in human ESCs. Analysis was performed with one-way ANOVA and Bonferroni's post hoc test. $* P<0.05, * \star \star P<0.001$. A full color version of this figure is available at https://doi.org/10.1530/JOE-20-0479.

and C). However, NE administered during the early pregnancy had no effect on the number of embryos (Fig. 5D). Further, embryonic development was observed on GD 13.5. These results showed that the average weights of embryos and placenta were significantly lower in mice injected with NE than that in the control mice (Fig. 5E, F and $G$ ), whereas no significant difference was observed in the number of absorbed embryos between the two groups (Fig. 5H). These results demonstrated that NE exposure inhibited embryonic growth.

\section{Discussion}

Women with excessive stress have reduced fertility and abnormal embryonic development; however, the underlying mechanism remains unknown. Under stress, after activating the sympathetic nervous system, the adrenal medulla synthesizes and secretes NE, which plays a biological role by binding to adrenergic receptors (ARs) on target cells and mainly results in vasoconstriction, increased blood pressure, accelerated heart rate, and excitable heart (Tank \& Lee Wong 2015, Pelliccia et al. 2017, Levy et al. 2018). NE is a catecholamine that functions both as a neurotransmitter and a hormone (Chrousos 2009). Previous studies on excess NE mainly focused on arrhythmias, generalized anxiety disorder, and cancer (Gellersen \& Brosens 2014, Gardner et al. 2016, Ricon et al. 2019). Endometrial ARs were observed to be expressed on stromal cells in decidualization. It implied that endometrium could be regulated by NE. The NE effect on endometrial decidualization has not yet been reported. In this study, NE was found to restrain uterine decidualization in early pregnancy. This study focused on the effect of NE on endometrial decidualization and showed that NE inhibited uterine decidualization by activating the PKC signaling pathway via $\alpha 1 \mathrm{~b}-\mathrm{AR}$. This study will provide 

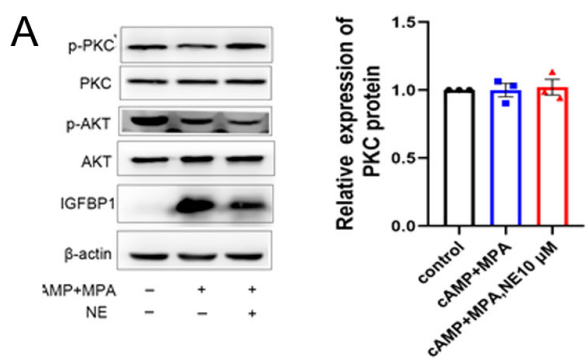

B
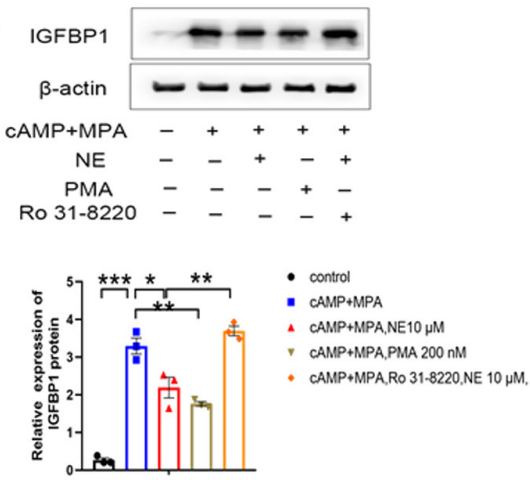
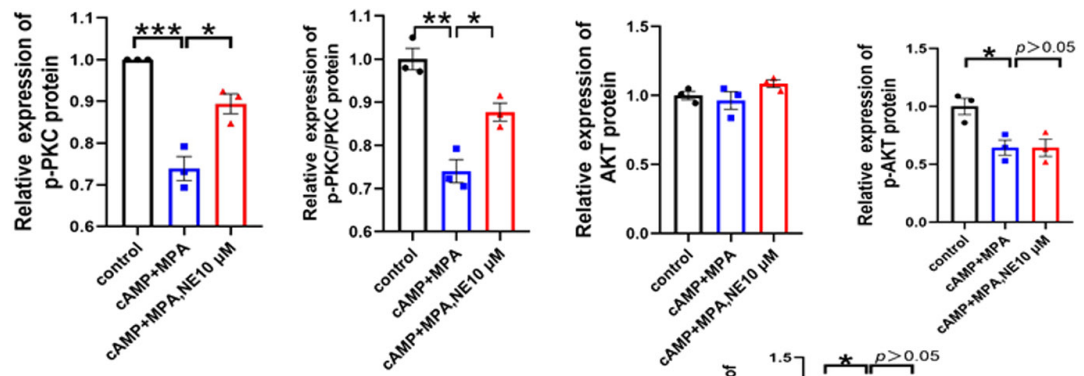

C
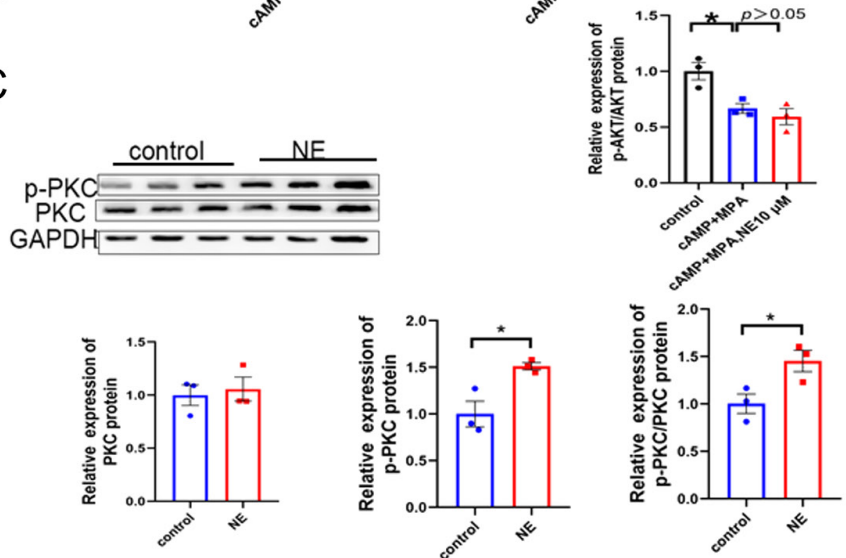

\section{Figure 4}

NE inhibits decidualization by activating the PKC signaling pathway. (A) PKC, p-PKC, AKT and p-AKT levels in ESCs treated with or without cAMP, MPA and NE were detected by Western blot. Analysis was performed with one-way ANOVA and Bonferroni's post hoc test. * $P<0.05$, $* \star P<0.01$. (B) Western blot analysis of the protein expressions of IGFBP1 in of ESCs pretreated with or without PKC pathway agonist (PMA, $200 \mathrm{nM}$ ) or inhibitor (Ro $31-8220,0.25$ $\mu \mathrm{M})$. Analysis was performed with one-way ANOVA and Bonferroni's post hoc test. ${ }^{*} P<0.05, * \star P<0.01, * \star \star P<0.001$. (C) The protein expressions of PKC and p-PKC of mice treated with or without NE daily for 3 days were detected by Western blot. Data are the means \pm S.E.M., $n=3, * P<0.05, * \star P<0.01$, $\star \star * P<0.001$. A full color version of this figure is available at https://doi.org/10.1530/JOE-20-0479.

novel conception for the prevention and treatment of stress-induced reproductive disorders.

Decidualization is a crucial event in early pregnancy. After implantation, ESCs begin to undergo decidualization for subsequent development, placentation, and growth of the embryo. Improper decidualization can trigger pathological changes and lead to adverse pregnancy outcomes (Patterson et al. 2017, Chen et al. 2019, Zheng et al. 2020). The mouse model of artificial decidualization demonstrated that NE could downregulate related markers and affect uterine decidualization. In vitro, NE was also found to inhibit decidual markers of human ESCs induced by cAMP and MPA.

To explore the mechanism of NE in inhibiting human ESCs decidualization, omics screening and GSEA analyses were performed. These results showed a significant enrichment in neuroactive ligand-receptor interactions of NE treatment group. ARs include several subtypes, and different intracellular signaling pathways are activated when NE binds with these different subtypes (Bylund et al. 1994). Previous studies have shown that the endometrium expresses $\alpha 1 b-A R, \alpha 2 b-A R$, and $\beta 2-A R$ (Wu et al. 2019). Western blots suggested that the $\alpha 1 \mathrm{~b}-\mathrm{AR}$ expression level increased after the NE treatment during decidualization of ESCs in vitro. Similarly, $\alpha 1 b$-AR was activated by NE during endometrial decidualization in vivo. To confirm the role of $\alpha 1 b-A R, \alpha 1 b-A R$ inhibitor, $\alpha 1 b-A R$ siRNA, and $\alpha 1 b-A R$ overexpression plasmid transfection technology with ESCs were used. Results of both approaches suggested that activation of $\alpha 1 b-A R$ may play an important role on NE effects. However, whether NE can directly bind to $\alpha 1 b-A R$ of ESCs still needs further exploration. In summary, NE could activate $\alpha 1 b-A R$ and inhibited endometrial decidualization both in vivo and in vitro.

In the PKC system, various hormones, neurotransmitters, etc. bind to receptors of cell membrane to activate phospholipase C $\beta$ (PLC), which decompose phosphatidylinositol biphosphate (PIP2) into diacylglycerol (DAG) and 1,4,5-inositol triphosphate (IP3). They act as second messengers and then trigger a series of intracellular reactions (Newton 2018). Previous studies revealed that PKC $\alpha$ was present in the deciduoma, and the PKC $\alpha$ signaling pathway was inhibited during the endometrial decidualization in rats (Shyu et al. 1997a,b, Liu et al. 1998). Similarly, we found p-PKC $\alpha$ expression was downregulated after decidualization was induced in 


\begin{tabular}{l|l|l|r|r|}
$\begin{array}{l}\text { Journal of } \\
\text { Endocrinology }\end{array}$ & J Wang et al. & $\begin{array}{l}\text { Norepinephrine regulates } \\
\text { endometrial decidualization }\end{array}$ & $\mathbf{2 4 8 : 3}$ & $\mathbf{2 8 5}$ \\
\hline
\end{tabular}

A

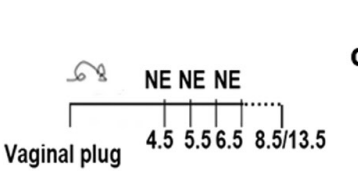

B

contro
NE

GD8.5

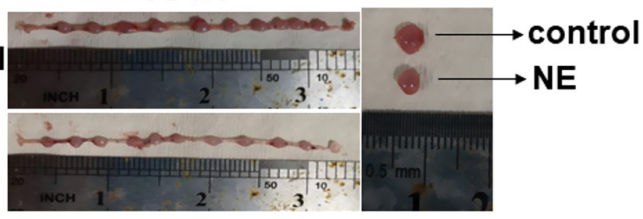

C

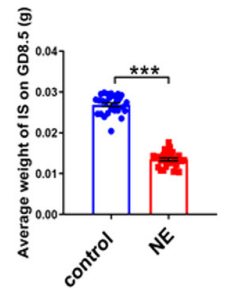

D

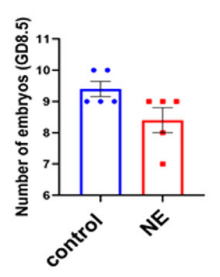

$\mathrm{H}$
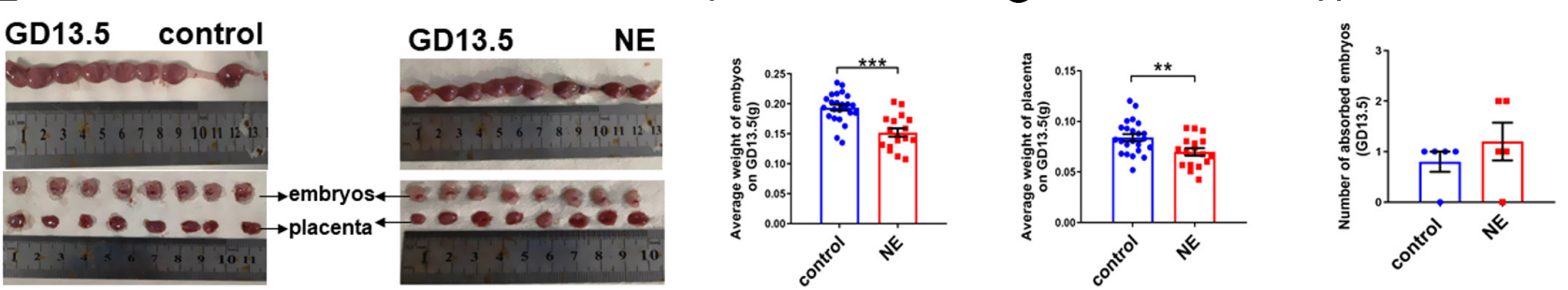

GD13.5

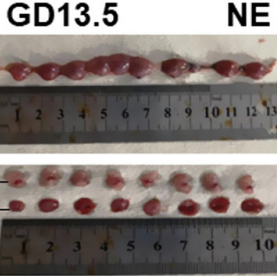

F

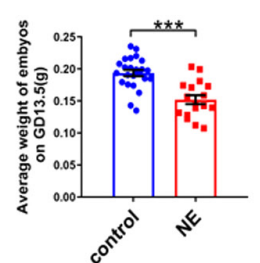

G

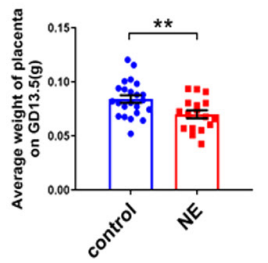

Figure 5

NE inhibits embryonic development in early pregnancy mice. (A) NE administration schedules of post-implantation mice. (B) Representative images of embryos in the uterus from post-implantation mice injected with PBS or NE on GD 8.5. (C) The average weight of IS was decreased in mice injected with PBS or NE on GD 8.5. IS, implantation site. Analysis was performed with Student's unpaired two-tailed $t$-test. *** $P<0.001$. (D) The number of embryos on GD 8.5. Analysis was performed with Chi-square test. (E) Representative images of embryos in the uterus from post-implantation mice injected with PBS or NE on GD 13.5. The average weight of embryos (F) and planta (G) was decreased in mice injected with PBS or NE on GD 13.5. Analysis was performed with Student's unpaired two-tailed $t$-test. $* \star P<0.01, * \star \star P<0.001(\mathrm{H})$ The number of absorbed embryos on GD 13.5. Analysis was performed with Chi-square test. ${ }^{* *} P<0.01,{ }^{* \star *} P<0.001$. A full color version of this figure is available at https://doi.org/10.1530/JOE-20-0479.

human ESCs, and activating the PKC signaling pathway with PMA inhibited this decidualization. Because the PKC signaling pathway is activated when NE upregulates to a1b-AR (Wang \& Ashraf 1998), we explored the effects of $\mathrm{NE}$ on the PKC signaling pathway in decidualized ESCs. Results showed that the p-PKC not total PKC expression level was significantly upregulated after the NE treatment both in vitro and in vivo, suggesting $\mathrm{NE}$ activated PKC signaling pathway. Furthermore, Pretreatment with Ro318220, a PKC pathway inhibitor, reverted the inhibitory effect of NE on decidualization. These data indicated NE inhibited endometrial decidualization by activating the PKC signaling pathway.

Unlike the PKC system, extracellular signals of PKA system are combined with corresponding receptors to induce intracellular responses by regulating the level of the second messenger cAMP. Previous studies showed that p-AKT was inhibited in endometrial decidualization (Wei et al. 2018), but the effect of NE on the PKA signaling pathway was still unclear. Neha J Patel found that NE could promote cell survival signaling in hippocampal neurons, significantly upregulated, whereas PI3K/Akt inhibitor LY294002 significantly downregulated p-Akt immunoreactivity with respect to vehicle-treated controls (Patel et al. 2010). However, Mar Coll demonstrated Droxidopa, an oral norepinephrine precursor, improved

(C) 2021 Society for Endocrinology Published by Bioscientifica Ltd. Printed in Great Britain hemodynamic and renal alterations of portal hypertensive rats, Droxidopa-treated rats showed a decreased ratio of p-AKT/AKT in superior mesenteric artery (Coll et al. 2012). In our experiment, we also found that the expression of p-AKT was significantly downregulated in decidualized ESCs induced by cAMP and MPA, but $10 \mu \mathrm{M}$ NE couldn't increase the downregulated expression of p-AKT.

Using a normal pregnancy model, the size and weight of the embryo implantation site in the NE-treated group were found to be significantly lower than those in the control group on GD 8.5. Moreover, the weights of embryos and placentas in the NE-treated group were significantly lower than that in the control group on GD 13.5. These results suggested that NE exposure inhibited the growth and development of embryos and fetuses. This study provides new ideas for the clinical prevention and treatment of endometrial decidualization and intrauterine growth restriction caused by excessive stress. It is well-known that decidualization of the mouse endometrium occurs after embryo implantation (GD 4.5). In our experiment, we injected NE intraperitoneally after embryo implantation (GD 5.5-7.5) to avoid the direct impact of NE on embryo implantation. That is why there is no significant difference of embryo implantation numbers between NE treatment and the control group. 


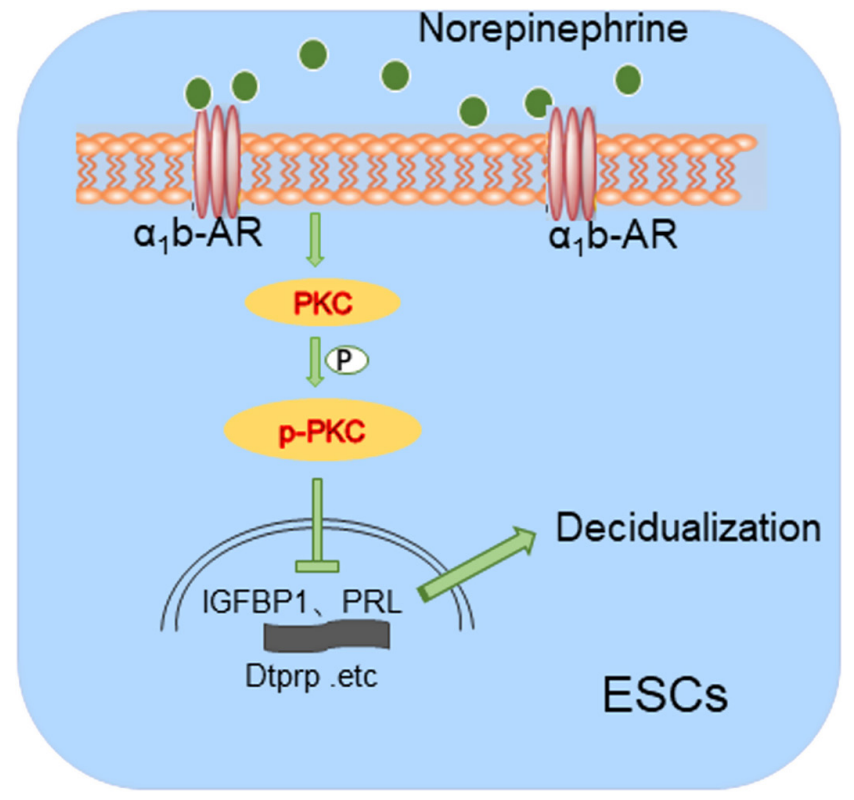

Figure 6

Schema of how NE/ 1 b-AR/PKC signaling impairs uterine decidualization. In decidualization, NE activated $\alpha 1 \mathrm{~b}-\mathrm{AR}$ on the cell membrane of ESCS, and then the intracellular PKC was phosphorylated, inhibiting the expression of IGFBP1, PRL, Dtprp, etc. related to cell differentiation. A full color version of this figure is available at https://doi.org/10.1530/ JOE-20-0479.

Excessive stress leads to a series of abnormal reproductive events, mechanisms that have not been elucidated. This study analyzed the effects of NE (one of the main stress hormones) on endometrial decidualization. However, identifying the precise mechanisms of longterm chronic or acute stress on decidualization in early pregnancy requires further experimental research.

In conclusion, NE inhibited endometrial decidualization of the endometrium through the activation of the PKC signaling pathway by upregulating $\alpha 1 b-A R$ (Fig. 6). Our results partially revealed potential molecular stress pathways that affect endometrial decidualization.

\section{Declaration of interest}

The authors declare that there is no conflict of interest that could be perceived as prejudicing the impartiality of the research reported.

\section{Funding}

This work was supported by the National Key R\&D Program of China (2017YFC1001403, 2017YFC1001404), National Natural Science Foundation of China (31970859, 81630036, 91542116), The Strategic Collaborative Research Program of the Ferring Institute of Reproductive Medicine Supported by Ferring Pharmaceuticals and Chinese Academy of Sciences (FIRMX200504), the Innovationoriented Science and
Technology Grant from NHC Key Laboratory of Reproduction Regulation (CX20172) and Innovative research team of high-level local universities in Shanghai.

\section{Author contribution statement}

Meirong Du conceived the study; Dajin Li, Meirong Du and Jiju Wang designed experiments; Jiju Wang performed research, acquired data and drafted the manuscript; Songcun Wang helped to perform experiments and analyze data; Yunhui Tang and Liyuan Cui helped collecting the samples and interpreted data; Dajin Li and Meirong Du revised the manuscript; all authors gave the final approve of the version to be published.

\section{References}

Abdullah LH, Bundy JT, Ehre C \& Davis CW 2003 Mucin secretion and PKC isoforms in SPOC1 goblet cells: differential activation by purinergic agonist and PMA. American Journal of Physiology: Lung Cellular and Molecular Physiology 285 L149-L160. (https://doi. org/10.1152/ajplung.00359.2002)

Anders S \& Huber W 2010 Differential expression analysis for sequence count data. Genome Biology 11 R106. (https://doi.org/10.1186/ gb-2010-11-10-r106)

Andrade CG, Abrahamsohn PA, Godinho F, Samuel E \& Zorn TM 1996 Death and replacement of uterine epithelial cells during oil-induced deciduoma development in the mouse. Anatomical Record 244 316-326. (https://doi.org/10.1002/(SICI)10970185(199603)244:3<316::AID-AR4>3.0.CO;2-W)

Besson B, Kim S, Kim T, Ko Y, Lee S, Larrous F, Song J, Shum D, Grailhe R \& Bourhy H 2019 Kinome-wide RNA interference screening identifies mitogen-activated protein kinases and phosphatidylinositol metabolism as key factors for rabies virus infection. mSphere 4. (https://doi.org/10.1128/mSphere.00047-19)

Brosens JJ \& Gellersen B 2006 Death or survival - progesteronedependent cell fate decisions in the human endometrial stroma. Journal of Molecular Endocrinology 36 389-398. (https://doi. org/10.1677/jme.1.02060)

Buzzio OL, Lu Z, Miller CD, Unterman TG \& Kim JJ 2006 FOXO1A differentially regulates genes of decidualization. Endocrinology 147 3870-3876. (https://doi.org/10.1210/en.2006-0167)

Bylund DB, Eikenberg DC, Hieble JP, Langer SZ, Lefkowitz RJ, Minneman KP, Molinoff PB, Ruffolo RR, Jr \& Trendelenburg U 1994 International union of pharmacology nomenclature of adrenoceptors. Pharmacological Reviews 46 121-136.

Chen W, Lu S, Yang C, Li N, Chen X, He J, Liu X, Ding Y, Tong C, Peng C et al. 2019 Hyperinsulinemia restrains endometrial angiogenesis during decidualization in early pregnancy. Journal of Endocrinology 243 137-148. (https://doi.org/10.1530/JOE-19-0127)

Chrousos GP 2009 Stress and disorders of the stress system. Nature Reviews: Endocrinology 5 374-381. (https://doi.org/10.1038/ nrendo.2009.106)

Coll M, Rodriguez S, Raurell I, Ezkurdia N, Brull A, Augustin S, Guardia J, Esteban R, Martell M \& Genescà J 2012 Droxidopa, an oral norepinephrine precursor, improves hemodynamic and renal alterations of portal hypertensive rats. Hepatology 56 1849-1860. (https://doi.org/10.1002/hep.25845)

Eyal O, Jomain JB, Kessler C, Goffin V \& Handwerger S 2007 Autocrine prolactin inhibits human uterine decidualization: a novel role for prolactin. Biology of Reproduction 76 777-783. (https://doi. org/10.1095/biolreprod.106.053058)

Frazier T, Hogue CJR, Bonney EA, Yount KM \& Pearce BD 2018 Weathering the storm; a review of pre-pregnancy stress and risk of 
spontaneous abortion. Psychoneuroendocrinology 92 142-154. (https:// doi.org/10.1016/i.psyneuen.2018.03.001)

Gardner RT, Ripplinger CM, Myles RC \& Habecker BA 2016 Molecular mechanisms of sympathetic remodeling and arrhythmias. Circulation: Arrhythmia and Electrophysiology 9 e001359. (https://doi.org/10.1161/ CIRCEP.115.001359)

Gellersen B \& Brosens JJ 2014 Cyclic decidualization of the human endometrium in reproductive health and failure. Endocrine Reviews 35 851-905. (https://doi.org/10.1210/er.2014-1045)

Kimura F, Takakura K, Takebayashi K, Ishikawa H, Kasahara K, Goto S \& Noda Y 2001 Messenger ribonucleic acid for the mouse decidual prolactin is present and induced during in vitro decidualization of endometrial stromal cells. Gynecological Endocrinology 15 426-432. (https://doi.org/10.1080/gye.15.6.426.432)

Levy B, Clere-Jehl R, Legras A, Morichau-Beauchant T, Leone M, Frederique G, Quenot JP, Kimmoun A, Cariou A, Lassus J et al. 2018 Epinephrine versus norepinephrine for cardiogenic shock after acute myocardial infarction. Journal of the American College of Cardiology $\mathbf{7 2}$ 173-182. (https://doi.org/10.1016/j.jacc.2018.04.051)

Li Q, Kannan A, Wang W, Demayo FJ, Taylor RN, Bagchi MK \& Bagchi IC 2007 Bone morphogenetic protein 2 functions via a conserved signaling pathway involving Wnt4 to regulate uterine decidualization in the mouse and the human. Journal of Biological Chemistry 282 31725-31732. (https://doi.org/10.1074/jbc. M704723200)

Li MQ, Hou XF, Lv SJ, Meng YH, Wang XQ, Tang CL \& Li DJ 2011 CD82 gene suppression in endometrial stromal cells leads to increase of the cell invasiveness in the endometriotic milieu. Journal of Molecular Endocrinology 47 195-208. (https://doi.org/10.1530/JME-10-0165)

Li Q, Kannan A, Das A, Demayo FJ, Hornsby PJ, Young SL, Taylor RN, Bagchi MK \& Bagchi IC 2013 WNT4 acts downstream of BMP2 and functions via $\beta$-catenin signaling pathway to regulate human endometrial stromal cell differentiation. Endocrinology 154 446-457. (https://doi.org/10.1210/en.2012-1585)

Liu JY, Shyu JC, Chang CL, Tsai CC, Chang AC, Yang LC, Lin LY \& Hsieh YS 1998 Protein kinase C isoforms during the development of deciduomata in pseudopregnant rats. Life Sciences $6 \mathbf{3} 721-730$ (https://doi.org/10.1016/s0024-3205(98)00327-0)

Liu L, Huang X, Xu C, Chen C, Zhao W, Li D, Li L, Wang L \& Du M 2020 Decidual CD8(+)T cells exhibit both residency and tolerance signatures modulated by decidual stromal cells. Journal of Translational Medicine 18 221. (https://doi.org/10.1186/s12967-020-02371-3)

Mantena SR, Kannan A, Cheon YP, Li Q, Johnson PF, Bagchi IC \& Bagchi MK 2006 C/EBPbeta is a critical mediator of steroid hormone-regulated cell proliferation and differentiation in the uterine epithelium and stroma. PNAS 103 1870-1875. (https://doi. org/10.1073/pnas.0507261103)

Murata S, Taniguchi T \& Muramatsu I 1999 Pharmacological analysis of the novel, selective alpha1-adrenoceptor antagonist, KMD-3213, and its suitability as a tritiated radioligand. British Journal of Pharmacology 127 19-26. (https://doi.org/10.1038/sj.bjp.0702489)

Murata H, Tanaka S, Tsuzuki-Nakao T, Kido T, Kakita-Kobayashi M, Kida N, Hisamatsu Y, Tsubokura H, Hashimoto Y, Kitada M et al. 2020 The transcription factor HAND2 up-regulates transcription of the IL15 gene in human endometrial stromal cells. Journal of Biological Chemistry 295 9596-9605. (https://doi.org/10.1074/jbc. RA120.012753)

Newton AC 2018 Protein kinase C: perfectly balanced. Critical Reviews in Biochemistry and Molecular Biology 53 208-230. (https://doi.org/10.108 0/10409238.2018.1442408)

Ng SW, Norwitz GA, Pavlicev M, Tilburgs T, Simón C \& Norwitz ER 2020 Endometrial decidualization: the primary driver of pregnancy health. International Journal of Molecular Sciences 21. (https://doi.org/10.3390/ ijms21114092)

Park Y, Nnamani MC, Maziarz J \& Wagner GP 2016 Cis-regulatory evolution of forkhead box O1 (FOXO1), a terminal selector gene for decidual stromal cell identity. Molecular Biology and Evolution 33 3161-3169. (https://doi.org/10.1093/molbev/msw193)

Patel NJ, Chen MJ \& Russo-Neustadt AA 2010 Norepinephrine and nitric oxide promote cell survival signaling in hippocampal neurons. European Journal of Pharmacology 633 1-9. (https://doi.org/10.1016/j. ejphar.2010.01.012)

Patterson AL, Pirochta J, Tufano SY \& Teixeira JM 2017 Gain-offunction beta-catenin in the uterine mesenchyme leads to impaired implantation and decidualization. Journal of Endocrinology 233 119-130. (https://doi.org/10.1530/JOE-16-0502)

Pelliccia F, Kaski JC, Crea F \& Camici PG 2017 Pathophysiology of takotsubo syndrome. Circulation 135 2426-2441. (https://doi. org/10.1161/CIRCULATIONAHA.116.027121)

Ricon I, Hanalis-Miller T, Haldar R, Jacoby R \& Ben-Eliyahu S 2019 Perioperative biobehavioral interventions to prevent cancer recurrence through combined inhibition of $\beta$-adrenergic and cyclooxygenase 2 signaling. Cancer 125 45-56. (https://doi. org/10.1002/cncr.31594)

Salsano S, Quiñonero A, Pérez S, Garrido Gómez T, Simón C \& Dominguez F 2017 Dynamic expression of PGRMC1 and SERBP1 in human endometrium: an implication in the human decidualization process. Fertility and Sterility 108 832-842.e1. (https://doi. org/10.1016/j.fertnstert.2017.07.1163)

Schatz F, Guzeloglu-Kayisli O, Arlier S, Kayisli UA \& Lockwood CJ 2016 The role of decidual cells in uterine hemostasis, menstruation, inflammation, adverse pregnancy outcomes and abnormal uterine bleeding. Human Reproduction Update 22 497-515. (https://doi. org/10.1093/humupd/dmw004)

Shyu JC, Hsieh YS, Chang CL, Tsai CC, Cheng CK \& Liu JY 1997a Change in protein kinase $\mathrm{C}$ activity on day 5 of decidualization in pseudopregnant rats. Chinese Journal of Physiology 40 107-112.

Shyu JC, Hsieh YS, Chang CL, Tsai CC, Chang AC, Yang LC, Lin MT, Cheng MH \& Liu JY 1997b Localization of protein kinase C alpha and zeta during the decidualization in pseudopregnant rats. Chinese Journal of Physiology 40 243-247.

Subramanian A, Tamayo P, Mootha VK, Mukherjee S, Ebert BL, Gillette MA, Paulovich A, Pomeroy SL, Golub TR, Lander ES et al. 2005 Gene set enrichment analysis: a knowledge-based approach for interpreting genome-wide expression profiles. PNAS $\mathbf{1 0 2}$ 15545-15550. (https://doi.org/10.1073/pnas.0506580102)

Tabanelli S, Tang B \& Gurpide E 1992 In vitro decidualization of human endometrial stromal cells. Journal of Steroid Biochemistry and Molecular Biology 42 337-344. (https://doi.org/10.1016/09600760(92)90137-8)

Tamura I, Jozaki K, Sato S, Shirafuta Y, Shinagawa M, Maekawa R, Taketani T, Asada H, Tamura H \& Sugino N 2018 The distal upstream region of insulin-like growth factor-binding protein-1 enhances its expression in endometrial stromal cells during decidualization. Journal of Biological Chemistry 293 5270-5280. (https://doi. org/10.1074/jbc.RA117.000234)

Tank AW \& Lee Wong D 2015 Peripheral and central effects of circulating catecholamines. Comprehensive Physiology 5 1-15. (https://doi. org/10.1002/cphy.c140007)

Wang Y \& Ashraf M 1998 Activation of alpha1-adrenergic receptor during $\mathrm{Ca} 2+$ pre-conditioning elicits strong protection against $\mathrm{Ca} 2+$ overload injury via protein kinase $\mathrm{C}$ signaling pathway. Journal of Molecular and Cellular Cardiology 30 2423-2435. (https://doi.org/10.1006/ jmcc.1998.0802)

Wang YM \& Yang ZC 2007 The influence of terbutaline on VEGF gene expression in rat astrocytes after norepinephrine and burn serum induction. Zhonghua Shao Shang Za Zhi=Zhonghua Shaoshang Zazhi = Chinese Journal of Burns 23 346-348.

Wang W, Li Q, Bagchi IC \& Bagchi MK 2010 The CCAAT/enhancer binding protein beta is a critical regulator of steroid-induced mitotic expansion of uterine stromal cells during decidualization. Endocrinology 151 3929-3940. (https://doi.org/10.1210/en.2009-1437) https://joe.bioscientifica.com

https://doi.org/10.1530/JOE-20-0479 (c) 2021 Society for Endocrinology Published by Bioscientifica Ltd. Printed in Great Britain 
Wang SF, Chen XH, He B, Yin DD, Gao HJ, Zhao HQ, Nan N, Guo SG Liu JB, Wu B et al. 2019 Acute restraint stress triggers progesterone withdrawal and endometrial breakdown and shedding through corticosterone stimulation in mouse menstrual-like model. Reproduction 157 149-161. (https://doi.org/10.1530/REP-18-0163)

Wei M, Gao Y, Lu B, Jiao Y, Liu X, Cui B, Hu S, Sun L, Mao S, Dong J et al. 2018 FKBP51 regulates decidualization through Ser473 dephosphorylation of AKT. Reproduction 155 283-295. (https://doi. org/10.1530/REP-17-0625)
Wu J, Kong S, Guo C, Wang J, Lu J, Jiang R \& Wang H 2019 An exaggerated epinephrine-adrenergic receptor signaling impairs uterine decidualization in mice. Reproductive Toxicology 90 109-117. (https://doi.org/10.1016/j. reprotox.2019.09.003)

Zheng HT, Fu T, Zhang HY, Yang ZS, Zheng ZH \& Yang ZM 2020 Progesterone-regulated Hsd11b2 as a barrier to balance mouse uterine corticosterone. Journal of Endocrinology 244 177-187. (https://doi. org/10.1530/JOE-19-0349)

Received in final form 15 December 2020

Accepted 5 January 2021

Accepted Manuscript published online 8 January 2021
(C) 2021 Society for Endocrinology Published by Bioscientifica Ltd. 